\title{
Successful joint ventures of plants: arbuscular mycorrhiza and beyond
}

\author{
Flavia Ercolin and Didier Reinhardt \\ Department of Biology, University of Fribourg, $\mathrm{CH}-1700$ Fribourg, Switzerland
}

\begin{abstract}
Among the oldest symbiotic associations of plants are arbuscular mycorrhiza (AM) with fungi of the phylum Glomeromycota. Although many of the symbiotic signaling components have been identified on the side of the plant, AM fungi have long evaded genetic analysis owing to their strict biotrophy and their exceptional genetics. Recently, the identification of the fungal symbiosis signal (Myc factor) and of a corresponding Myc factor receptor, and new insights into AM fungal genetics, have opened new avenues to address early communication and functional aspects of AM symbiosis. These advances will pave the way for breeding programs towards adapted AM fungi for crop production, and will shed light on the ecology and evolution of this remarkably successful symbiosis.
\end{abstract}

\section{Plants: symbiotic from the start}

According to the endosymbiont hypothesis, the success of early eukaryotes was fostered by the acquisition of prokaryotic cells that evolved to cellular power plants, the mitochondria [1]. Such improvement of eukaryotes continued with the incorporation of photosynthetic prokaryotes, the ancestors of chloroplasts in plants [1]. After having radiated in aquatic environments, plants started to conquer the inhospitable expanses of the Ordovician approximately 475 million years (Myr) ago [2]. This transition coincided with the emergence of intracellular fungal interactions which might have been instrumental for early plants to cope with the harsh environmental conditions on land [3-5]. While mitochondria and chloroplasts are meanwhile regarded as integral constituents of plant cells, the fungal interaction is known as arbuscular mycorrhiza (AM), the most widespread symbiosis of plants [6]. Approximately 400 Myr later, the nitrogen-fixing root nodule symbiosis (RNS) with rhizobacteria (rhizobia) evolved in a subset of the dicotyledonous angiosperms (mostly legumes). Hence, cooperation has been a recurrent theme in the success story of plants and continues to enable them to inhabit various extreme environments $[7,8]$.

Similar to mitochondria and chloroplasts, AM fungi and rhizobia are accommodated within the cytoplasm of their host, a condition which facilitates the exchange of nutrients and signal molecules, but requires specific adaptations [9]. The understanding of mechanisms involved in the establishment of AM and RNS has in recent years seen dramatic advances through genetic analysis of the plant host [6]. On the microbial side, the role of rhizobia in RNS

Corresponding author: Reinhardt, D. (didier.reinhardt@unifr.ch). has been characterized in considerable detail, whereas AM fungi have long evaded genetic analysis, owing to their strictly biotrophic lifestyle, their coenocytic organization, their heterokaryotic nature and their asexual mode of propagation [10]. A central open question has been how the apparent involvement of symbiotic communication in $\mathrm{AM}$ can be reconciled with the very low host specificity in this symbiosis [9]. Furthermore, it has been a challenge for evolutionary theory to explain how AM fungi as ancient asexuals could have persisted and thrived over hundreds of millions of years without negative effects on their own fitness and on the evolution of AM symbiosis [3,11]. In this opinion article, we discuss recent findings that provide a basis to address these questions and we consider how the exceptional genetics of AM fungi might have shaped the evolution and ecology of AM symbiosis. Despite the ancestral status of AM, we start with an overview over the better explored signaling mechanisms in RNS, which have much in common with signaling in AM.

\section{Symbiotic signaling: insights from nodulation}

For plants it is crucial to rapidly distinguish between the diverse microbes in the soil, potential symbionts from neutral epiphytes and pathogens. The RNS of most legumes is initiated by Nod factors (NFs) from rhizobia which are recognized as symbiosis signals by LysM-type Nod factor receptors (NFRs) in root hairs [12] (Figure 1). NFs are lipochitooligosaccharides (LCOs) that consist of an $N$-acetylglucosamine backbone with various substitutions, which are the basis for the wide diversity of NFs [13]. Each rhizobial strain produces one or several characteristic NFs, which require particular NFRs for recognition in the plant, thus contributing to the restricted host range in RNS [13,14]. A signaling cascade relates NF perception from the plasma membrane to the nucleus and triggers a symbiotic program that leads to intracellular accommodation of the endosymbiont $[6,15,16]$. A central component of symbiotic signaling is an oscillatory calcium signal (calcium spiking) which is thought to be perceived by a calcium and calmodulin-dependent protein kinase (CCaMK). CCaMK is a pivotal element in Symbiosis (SYM) signaling, since its activation is necessary and sufficient to trigger nodule development in legumes in the absence of any upstream signal [17-20]. AM symbiosis requires several of the $S Y M$ genes identified in RNS, which are therefore referred to as 'common SYM genes' (Figure 1). Because the evolution of AM preceded the emergence of nodulation by several hundred million years, it appears that the common SYM pathway evolved in the context of AM and became secondarily involved in RNS $[4,6]$. 


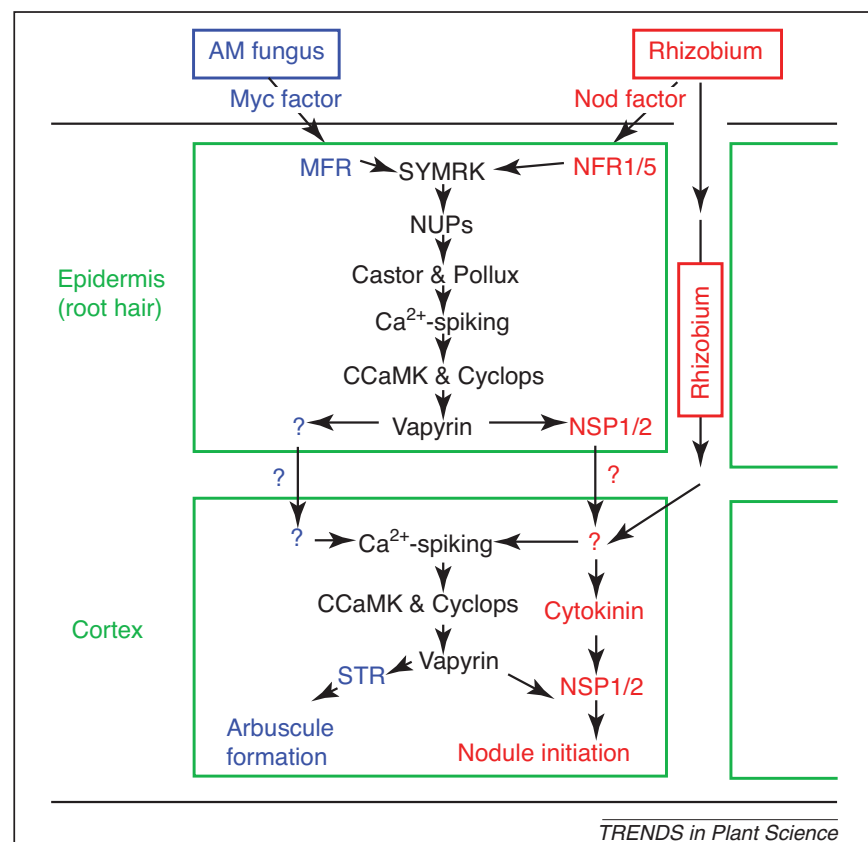

Figure 1. Symbiotic signaling in AM and RNS. AM-related signaling components are depicted in blue, RNS-related components are depicted in red, common signaling components are depicted in black. Rhizobia produce NFs that are recognized by Nod factor receptor 1 (NFR1) and NFR5, whereas AM fungi release Myc factors that are recognized by Myc factor receptors (MFRs). Second messengers from NFRs and MFRs are integrated by symbiosis receptor kinase (SYMRK) and transduced through the common SYM pathway. Successful intracellular accommodation in epidermal cells requires VAPYRIN and, in the case of RNS, the GRAS-type transcription factors NODULATION SIGNALING PATHWAY 1 (NSP1) and NSP2. In the case of RNS, a mobile signal is generated that triggers organogenesis in the cortex through cytokinin. Rhizobia can also infect intercellularly through the apoplast under conditions which prevent signaling through the common SYM pathway. Invasion of cortical cells requires the common SYM genes that operate downstream of calcium spiking including CCAMK, CYCLOPS and VAPYRIN, whereas STUNTED ARBUSCULE (STR) is involved specifically in AM. Note that only the core components discussed here are depicted. Question marks indicate unknown components that link epidermal events with cortical stages.

The canonical infection pathway through root hairs is by no means the only entry path for rhizobia into legume roots. In Sesbania rostrata and other tropical legumes, rhizobia can invade intercellularly through openings in the root surface, which can result from lateral root emergence [21]. Interestingly, this alternative way of entry, referred to as 'crack entry', which is favored when plants are waterlogged, does not require some upstream components of the common SYM pathway (Figure 1), and exhibits relaxed signaling requirements compared to root hair infection [22]. Furthermore, it requires ethylene, which normally inhibits rhizobial infection through root hairs [23]. Interestingly, Lotus japonicus mutants with a defect in a common $S Y M$ gene can be infected by rhizobia through an ethylene-dependent intercellular pathway [24], consistent with the (re)activation of the crack entry pathway, which is regarded as a primitive infection mechanism that preceded the evolution of root hair infection [15].

In some cases, rhizobia can infect root or stem tissues intercellularly without the involvement of NFs or common SYM signaling [25]. In these cases, purine derivatives can serve as symbiotic signals for nodule primordium formation. In this context, it should be noted that nitrogen-fixing bacteria in grasses can be hosted in root tissues in the absence of accommodation structures such as nodules [26]. Taken together, these observations suggest a stepwise evolution of RNS [15,27]. Initially, nodule formation and intracellular accommodation of bacteria could have evolved from purely intercellular interactions that relied on crack entry. Subsequently, the intercellular infection pathway could have been replaced by the more efficient and more stringent intracellular infection pathway through root hairs [15]. This route of infection efficiently circumvents the epidermal barrier without the need for openings in the root surface. However, the older intercellular infection pathway is still operational, and can be used when signaling through the common SYM pathway is impaired [22,24].

Interestingly, growth of nodule primordia in the root cortex is already triggered during root hair infection, that is well before rhizobia have reached the cortex, pointing to a mobile signal between epidermis and cortex [12]. The relationship between the events in root hairs and the cortex has long remained unclear because they could not be studied separately, owing to their common linkage to NF signaling. Only recently, these two aspects of RNS became disentangled, owing to dominant gain-of-function mutants in CCaMK, which generate nodules in the absence of rhizobia, hence allowing to genetically separate primordium formation from root hair infection $[18,19]$.

\section{Communication between plants and AM fungi and development of AM symbiosis}

AM fungi have long been known to release diffusible signal molecules (Myc factors) that trigger the expression of symbiosis-related genes such as ENOD11 [28]. The Myc factor (MF) of Glomus intraradices has recently been shown to consist of LCOs that are closely related to NFs [29]. Considering the relatively recent evolution of RNS, this suggests that LCOs evolved first as Myc factors in AM and became secondarily 'copied' as NFs in rhizobia. This also suggests similar perception mechanisms in the plant host. Indeed, one single LysM receptor kinase mediates recognition of both, rhizobia and AM fungi, in Parasponia andersonii (order Rosales) [30], pointing to either a common LCO signal or to limited specificity of the Parasponia LysM receptor kinase. In this context, it is interesting to note that an Arabidopsis NFR1 homolog (CERK1) is a receptor for chitin [31,32], which corresponds to the undecorated backbone of MFs and NFs. However, the NFRs of legumes are specific to RNS, hence it remains to be seen whether in legumes other LysM receptors might be responsible for MF perception. The fact that LysM-type receptor kinases are generally encoded by large gene families in AM-competent plants [33] indicates that plants could potentially perceive multiple different MFs, which would explain the broad host range in AM (see below).

As in RNS, the common signaling pathway is required for successful intracellular colonization of epidermal cells by AM fungi (Figure 1). Mutations in common SYM genes generally lead to an early arrest of hyphal infection [6]; however, AM fungi can in many cases overcome this barrier and later form normal arbuscules in the cortex [24,3436]. These observations indicate that SYM gene signaling is particularly crucial for epidermal infection. However, as in the case of RNS, downstream common $S Y M$ genes such 
Sexual reproduction entails considerable costs arising from the energy invested in the search for mating partners and in courtship behavior and from the fact that only half of the individuals in a given population produce offspring. Nevertheless, sexual reproduction is by far more common than asexual reproduction. This is thought to be as a result of the positive effects of sex, such as the generation of genetic diversity, the complementation of mutations, the elimination of deleterious alleles and the combination of advantageous alleles through recombination [69]. However, in a few cases, asexual lineages have survived for dozens or hundreds of millions of years [70].

AM fungi have existed for over $400 \mathrm{Myr}$ in a constant form and are thought to be ancient asexuals [11]. AM fungi are coenocytic, that is they have a continuous cytoplasm (without cross walls) in which thousands of nuclei coexist (Figure 2). These 'nuclear populations' encode surprisingly diverse genomes and several lines of evidence indicate that the nuclei themselves are diverse, that is AM fungi are heterokaryotic $[45,46]$. In fact, the genome space of AM fungi might be equivalent to more than ten different (but similar) nuclear genomes, which makes the Glomus sequencing project a major challenge [50].

as $C Y C L O P S$ and $C C a M K$ are indispensable for epidermal infection as well as for cortical infection and arbuscule formation (Figure 1). Whether colonization of the root cortex by AM fungi involves mobile signals as in RNS is unknown, but given the rapid intercellular progression of fungal hyphae from the epidermis towards the cortex such a signal might not be required.

Plants have evolved a cellular infection structure, the prepenetration apparatus (PPA), which guides AM fungi through epidermal cells [37] in a similar way to the infection thread in RNS [38]. Whether the rare infection events in the absence of SYM signaling involve a PPA is not clear at present. Arbuscule formation in cortical cells also involves PPA formation [39]. The fact that arbuscules can be formed in some sym mutants suggests that cortical PPAs can be triggered independently of SYM signaling or that PPA formation is not an absolute requirement for arbuscule formation. Unfortunately, the separate analysis of epidermal infection and arbuscule formation in the cortex is more difficult than genetic separation of epidermal and cortical events in RNS, where nodule primordium formation can be studied in the absence of the microbe (see above).

Recently, VAPYRIN has been discovered as a new component required for intracellular accommodation of AM fungi and rhizobia in epidermal, as well as cortical cells [40-42]. VAPYRIN consists of a major sperm protein domain and an ankyrin domain, both known to mediate protein-protein interactions. The large ankyrin domain with 11 repeats is unique in plants [43], and closely resembles animal ankyrins which serve to connect integral membrane proteins to elements of the spectrin cytoskeleton [44]. Based on this structural similarity, VAPYRIN might promote intracellular accommodation of endosymbionts by interacting with membranes and/or with the cytoskeleton. Indeed, VAPYRIN protein associates with small membranous compartments, the function of which remains to be established $[40,41]$. Thus, VAPYRIN and the identification of its potential interaction partners will provide an entry point to the elusive molecular mechanisms involved in intracellular accommodation of endosymbionts.
Theoretically, prolonged clonal expansion of a syncytium should result in increasing genetic heterogeneity owing to the accumulation of mutations, the deleterious consequences of which are buffered by complementation from other nuclei. By contrast, increasing heterogeneity should be counteracted by uneven segregation and loss of nuclear variants in the individual hyphae of the expanding syncytium (genetic drift; Figure 2).

AM fungi can reshuffle their genome by anastomosis, that is hyphal fusions that include the exchange of nuclei [71]. Anastomosis between genetically similar hyphae can potentially counteract the loss of nuclear variants, whereas anastomosis between genetically different hyphae generates new genetic diversity (Figure 2). Environmental factors can impose selection pressure on individual mycelia within a population of anastomosing AM fungi. In addition, selective promotion and sanctioning of the strictly biotrophic AM fungi by plants can potentially lead to selection of certain beneficial genotypes within a fungal population. Hence, the evolution of AM fungi might have been shaped to a considerable degree by their exceptional genetics.

\section{Genetic diversity of AM fungi: relevant for symbiotic signaling and functioning?}

AM fungi are exceptional owing to their supposed ancient asexual nature and their coenocytic organization [10]. In addition, several lines of evidence suggest that AM fungi are heterokaryotic, that is that they contain genetically different nuclei $[45,46]$ (Box 1). However, based on the observation that the degree of genetic heterogeneity remained unchanged over multiple generations, this view has been questioned and, instead, increased ploidy or gene duplication has been invoked to explain the genetic diversity within individual fungal isolates [47]. The apparent dilemma might result from hyphal fusions (anastomoses), which involve the exchange of nuclei (Box 1). Hence, in a heterokaryotic syncytium, the loss of genetic diversity through genetic drift could be counteracted by continuous reshuffling of the different nuclear variants [48] (Figure 2). In addition, maintenance of the heterokaryotic status might be enforced by selection for essential gene copies among the partially degenerated subgenomes, which are expected to result from prolonged clonal propagation. In practical terms, the unusual genetic organization of AM fungi makes forward genetic approaches and stable genetic transformation virtually impossible [49] and it renders the assembly of the $G$. intraradices genome an unprecedented challenge [50].

Could the genetic diversity of AM fungi be relevant for symbiotic communication and functioning of AM? In analogy to the rhizobial NOD genes which encode enzymes in NF biosynthesis [13], AM fungal genomes are likely to encode enzymes for MF biosynthesis (MYC genes). Similar to protein-coding genes such as the catalytic subunit of DNAPolymerase- $\alpha$ [47], MYC genes might have diversified to encode enzymes with different activities that produce a blend of diverse MFs, providing to AM fungi a broad signaling potential. Such a hypothetical diversification of MFs could have promoted the expansion and diversification of the LysM receptor kinase families in plants [33] in a process analogous to the coevolution of microbial avirulence genes and resistance genes in plant-pathogen interactions [51], with the difference that the outcome of recognition in a plant-pathogen interaction is incompatibility, whereas 


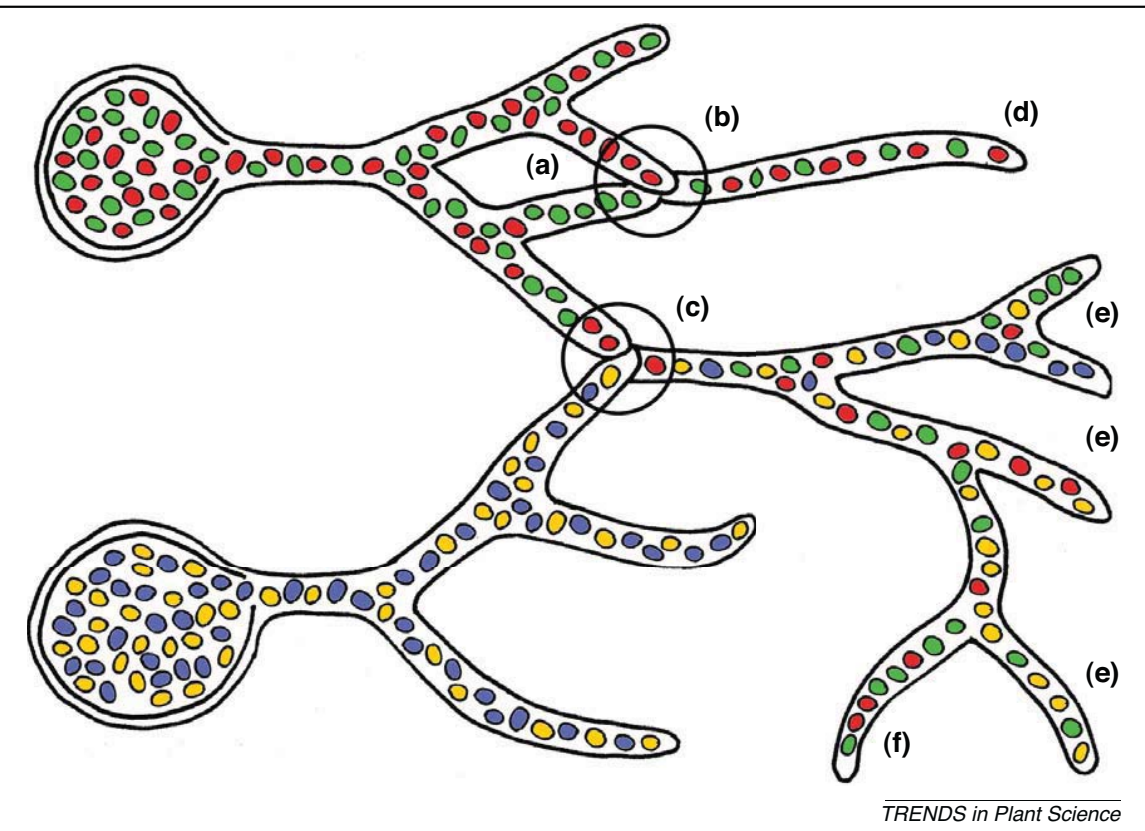

Figure 2. Parasexual processes in AM fungi. AM fungi are coenocytic, that is they lack cross walls in their hyphae and they are heterokaryotic, because they contain genetically different nuclei (represented by different colors). As a consequence of these features, AM fungi can be subject to genetic drift owing to uneven segregation of their nuclear variants (a). Fungal hyphae can fuse and exchange cytoplasm and organelles, including nuclei, in a process known as anastomosis. Anastomosis can occur between hyphae originating from the same clone (b) or from genetically different clones (c). In the first case, anastomosis can restore the original genetic diversity, thereby counteracting genetic drift (d). In the second case, new diversity is generated through the combination of different nuclear variants. In the course of subsequent clonal growth, new genetic combinations can be generated through genetic drift and/or selection (e) or the original genetic setup can be restored (f). Taken together, these mechanisms provide an alternative to chromosomal recombination in sexual reproduction.

the recognition in AM results in compatibility. Conceivably, recognition in AM confers a strong selective advantage to both symbiotic partners, providing a strong driving force for coevolution. Although the described scenario could explain the broad host range in AM, it remains to be seen whether AM fungi have the potential to produce more than a few MFs as in the case of $G$. intraradices [29]. Future biochemical analysis of MF composition in different AM fungal isolates (grown under various conditions) and functional analysis of LysM receptor kinases in plants will shed light on these issues.

Although AM fungal genetics are unusual and difficult to approach, genetic manipulation has been achieved using their parasexual mechanisms (Box 1). A considerable step ahead represents the recent characterization of crosses between genetically different strains of $G$. intraradices [52]. AM fungi cannot be grown in pure culture, but $G$. intraradices can be cultured in monoxenic systems with living roots as a substrate. Co-culturing genetically different fungal isolates resulted in new fungal hybrid lines that carried genetic markers of the two parent isolates [52]. Subsequent culture over several generations gave rise to segregating lines with new growth characteristics and, remarkably, with diverse symbiotic potential [53]. Hence, anastomosis can generate new diversity within AM fungal syncytia (Figure 2) and explains the occurrence of genetic recombination in natural AM fungal populations $[54,55]$. However, it should be pointed out that recombination in the context of AM fungi refers to the mixing and segregation of different nuclear types, although genetic recombination of chromosomes within the syncytium cannot be ruled out $[10,55]$. These results highlight a second level at which genetic diversity of AM fungi can impinge on AM symbiosis. Variability in the degree to which plants benefit from AM [56] is likely to depend on the genetic setup of both, the plant and its AM fungal partner. Conceivably, AM fungal genomes could encode a wide variety of functionally important proteins such as nutrient transporters. Such functional diversity could represent the substrate for adaptive evolution through selection by the plant host (see below).

\section{What keeps symbionts together?}

Mutual symbiosis has been predicted to be inherently unstable owing to conflict of interest [57]. For example, one partner can improve its own fitness by exploiting the other without returning any benefit (parasitism) or the interaction can simply decay if one partner ceases to interact and becomes free-living [58]. Unstable mutualism has indeed been observed in the case of ectomycorrhizal (ECM) fungi among the basidiomycetes. During their evolution, these ECM fungi have repeatedly gained and lost symbiotic capacity [59]. This observation is in line with the finding that ECM fungi have retained most genes required for saprotrophic life, except for cell wall-degrading enzymes [60]. Similarly, phylogenetic analysis of rhizobia showed that non-nodulating free-living strains were in several cases nested in clusters of nodulating strains, indicating that they had secondarily lost the ability to engage in RNS, whereas free-living strains have repeatedly gained symbiotic capacity [61]. This shows that in evolutionary terms, ECM fungi and rhizobia entertain more relaxed relationships with their hosts than AM fungi, which have never been reported to become free-living.

In contrast to RNS and ECM, AM symbiosis appears to have been remarkably stable throughout its long history of 


\section{Box 2. With a carrot and a stick: are plants dominating AM fungi?}

Plants invest carbohydrate equivalents into AM fungi and gain fitness in terms of improved mineral nutrition and increased tolerance to biotic and abiotic stresses $[9,72]$. To avoid fungal proliferation to an extent where the costs of the interaction would potentially exceed its benefits, plants can limit the degree of root colonization by AM fungi [73], potentially through reduction of carbohydrate allocation to the fungus. In addition, plants reduce the levels of AM fungal colonization under conditions where the interaction is not beneficial, for example when the plant is well supplied with mineral nutrients and therefore does not profit from symbiosis $[74,75]$, or under conditions when the symbiosis is rendered ineffective by mutation or downregulation of phosphate transporters $[64,65]$. These observations suggest that the plant has control over microbial growth in the root.

Given the strict biotrophy of AM fungi and the fact that plants can limit the extent of fungal colonization, are AM fungi at the mercy of their plant host? At the level of individual plants in pot cultures ('oneplant-one-fungus' situation), this might indeed be the case. However,

over 400 Myr [3,11]. The vast majority of plants form AM and all Glomeromycota are obligate symbionts [9]. What might have stabilized the association through evolution? In theory, mutualistic interactions can persist if one partner can preferentially chose the most beneficial partner and impose sanctions on cheaters. Indeed, such sanctions have been documented in RNS and in AM that had been rendered ineffective experimentally, either by mutation or by manipulation of the environment (Box 2). When rhizobia were prevented from fixing atmospheric nitrogen $\left(\mathrm{N}_{2}\right)$ either by mutation of Nif genes [62] or by replacing $\mathrm{N}_{2}$ by argon [63], nodules were smaller and bacterial reproduction was reduced. Analogously, rendering AM ineffective by downregulation or mutation of symbiosis-specific phosphate transporters of the plant causes significant reduction of AM colonization $[64,65]$. In all these cases, the plant appears to detect the lack of benefit and responds with suppression of microbial proliferation in the root. Notably, in a natural setting, plants that are colonized by spatially separated AM fungi can distinguish them based on their symbiotic performance and deliver resources preferentially to the better mutualist [66]. Considering the great functional variability in AM associations [56], plants are faced with a broad spectrum of potential interactors among which they can preferentially select effective mutualists and sanction ineffective ones, which provides a positive feedback mechanism that stabilizes symbiosis. A rather prosaic explanation for the persistence of AM fungi in symbiosis would be their strict biotrophy, which could be related to partial degeneration of the genome as a consequence of prolonged clonal growth (Box 1). This scenario would be reminiscent of some bacterial endosymbionts of insects, which have undergone reductive genome evolution and, as a consequence, are strictly biotrophic, including the irreversible loss of a freeliving state [67]. Deciphering the genome of $G$. intraradices and other AM fungi will address this question and will provide a starting point to understand the relevance of genetic diversity for evolution and ecology of AM.

\section{Concluding remarks}

The recent progress discussed here opens new directions to address fundamental aspects of AM symbiosis such as the determinants of host range and specificity, mechanisms involved in intracellular accommodation of the endosym- natural conditions are rather different. When a seed germinates in its natural habitat, AM fungi are already present and established in symbiosis with older plants; hence, they do not need the new seedling for survival. By contrast, the young plant is subject to intraand interspecific competition and can suffer from drought stress and nutrient starvation, conditions that can be alleviated by AM fungi $[76,77]$. In addition, in arid regions, young plants can profit from hydraulically lifted water from older plants, which is transferred through AM fungi [78].

Interestingly, AM colonization can result in a reduction of direct phosphate uptake capacity in the plant which is (over)compensated by fungal phosphate delivery $[79,80]$. This phenomenon might represent a mechanism by which the fungus renders the plant dependent on its services, thereby stabilizing compatibility. Taken together, depending on plant age, environmental conditions and the scale of observation, the plant might be at the mercy of the fungus, rather than vice versa.

biont and the genetic basis of its mutualistic potential. Although these issues seem at first to have little in common, they are linked. The genetic setup of AM fungi is likely to influence communication with their hosts and functionality of the established symbiosis. Further studies on the genetics and genomics of $G$. intraradices and other AM fungi will provide the tools to elucidate the significance of their genetic diversity for AM symbiosis. This knowledge can be used to breed new AM fungal strains adapted to crop plants under field conditions. In addition, it will provide insights into the factors that influence AM fungal ecology in natural settings and into the mechanisms that have allowed AM fungi to live their 'scandalous' life as ancient asexuals since hundreds of millions of years [68].

\section{Acknowledgments}

We thank C. Lexer and A. Buchala for critical reading of the manuscript and M. van der Heijden for helpful discussions. Our work is supported by a grant from the Swiss National Science Foundation grant to D.R (31003A-118055) and by the Swiss National Center of Competence in Research (NCCR) 'Plant Survival'.

\section{References}

1 Gray, M.W. (1999) Evolution of organellar genomes. Curr. Opin. Genet. Dev. 9, 678-687

2 Kenrick, P. and Crane, P.R. (1997) The origin and early evolution of plants on land. Nature 389, 33-39

3 Brundrett, M.C. (2002) Coevolution of roots and mycorrhizas of land plants. New Phytol. 154, 275-304

4 Kistner, C. and Parniske, M. (2002) Evolution of signal transduction in intracellular symbiosis. Trends Plant Sci. 7, 511-518

5 Redecker, D. et al. (2000) Glomalean fungi from the Ordovician. Science 289, 1920-1921

6 Parniske, M. (2008) Arbuscular mycorrhiza: the mother of plant root endosymbioses. Nat. Rev. Microbiol. 6, 763-775

7 Appoloni, S. et al. (2008) Molecular community analysis of arbuscular mycorrhizal fungi in roots of geothermal soils in Yellowstone National Park (USA). Microb. Ecol. 56, 649-659

8 Al-Yahya'ei, M. et al. (2011) Unique arbuscular mycorrhizal fungal communities uncovered in date palm plantations and surrounding desert habitats of Southern Arabia. Mycorrhiza 21, 195-209

9 Smith, S. and Read, D. (2008) Mycorrhizal Symbiosis, Academic Press

10 Sanders, I.R. and Croll, D. (2010) Arbuscular mycorrhiza: the challenge to understand the genetics of the fungal partner. Annu. Rev. Genet. 44, 271-292

11 Rosendahl, S. (2008) Communities, populations and individuals of arbuscular mycorrhizal fungi. New Phytol. 178, 253-266

12 Oldroyd, G.E.D. and Downie, J.A. (2008) Coordinating nodule morphogenesis with rhizobial infection in legumes. Annu. Rev. Plant Biol. 59, 519-546 
13 Denarie, J. et al. (1996) Rhizobium lipo-chitooligosaccharide nodulation factors: signaling molecules mediating recognition and morphogenesis. Annu. Rev. Biochem. 65, 503-535

14 Radutoiu, S. et al. (2007) LysM domains mediate lipochitinoligosaccharide recognition and $\mathrm{Nfr}$ genes extend the symbiotic host range. EMBO J. 26, 3923-3935

15 Charpentier, M. and Oldroyd, G. (2010) How close are we to nitrogenfixing cereals? Curr. Opin. Plant Biol. 13, 556-564

16 Oldroyd, G.E.D. et al. (2009) Reprogramming plant cells for endosymbiosis. Science 324, 753-754

17 Gleason, C. et al. (2006) Nodulation independent of rhizobia induced by a calcium-activated kinase lacking autoinhibition. Nature 441, 11491152

18 Hayashi, T. et al. (2010) A dominant function of CCaMK in intracellular accommodation of bacterial and fungal endosymbionts. Plant J. 63, 141-154

19 Madsen, L.H. et al. (2010) The molecular network governing nodule organogenesis and infection in the model legume Lotus japonicus. Nat. Commun. 1, 10

20 Tirichine, L. et al. (2006) Deregulation of a Ca2+/calmodulindependent kinase leads to spontaneous nodule development. Nature $441,1153-1156$

21 Goormachtig, S. et al. (2004) Switch from intracellular to intercellular invasion during water stress-tolerant legume nodulation. Proc. Natl. Acad. Sci. U.S.A. 101, 6303-6308

22 Capoen, W. et al. (2010) Sesbania rostrata: a case study of natural variation in legume nodulation. New Phytol. 186, 340-345

23 Gresshoff, P. et al. (2009) Genetic analysis of ethylene regulation of legume nodulation. Plant Signal. Behav. 4, 818-823

24 Groth, M. et al. (2010) NENA, a Lotus japonicus homolog of Sec13, is required for rhizodermal infection by arbuscular mycorrhiza fungi and rhizobia but dispensable for cortical endosymbiotic development. Plant Cell 22, 2509-2526

25 Giraud, E. et al. (2007) Legumes symbioses: absence of Nod genes in photosynthetic bradyrhizobia. Science 316, 1307-1312

26 Hurek, T. et al. (2002) Azoarcus grass endophytes contribute fixed nitrogen to the plant in an unculturable state. Mol. Plant-Microbe Interact. 15, 233-242

27 Sprent, J.I. (2007) Evolving ideas of legume evolution and diversity: a taxonomic perspective on the occurrence of nodulation. New Phytol. $174,11-25$

28 Kosuta, S. et al. (2003) A diffusible factor from arbuscular mycorrhizal fungi induces symbiosis-specific MtENOD11 expression in roots of Medicago truncatula. Plant Physiol. 131, 952-962

29 Maillet, F. et al. (2011) Fungal lipochitooligosaccharide symbiotic signals in arbuscular mycorrhiza. Nature 469, 58-64

30 Op den Camp, R. et al. (2011) LysM-type mycorrhizal receptor recruited for Rhizobium symbiosis in nonlegume Parasponia. Science 331, 909-912

31 Miya, A. et al. (2007) CERK1, a LysM receptor kinase, is essential for chitin elicitor signaling in Arabidopsis. Proc. Natl. Acad. Sci. U.S.A. 104, 19613-19618

32 Wan, J.R. et al. (2008) A LysM receptor-like kinase plays a critical role in chitin signaling and fungal resistance in Arabidopsis. Plant Cell 20, $471-481$

33 Zhang, X.C. et al. (2009) Evolutionary genomics of LysM genes in land plants. BMC Evol. Biol. 9, 183

34 Demchenko, K. et al. (2004) Distinct roles of Lotus japonicus SYMRK and SYM15 in root colonization and arbuscule formation. New Phytol. 163, 381-392

35 Kistner, C. et al. (2005) Seven Lotus japonicus genes required for transcriptional reprogramming of the root during fungal and bacterial symbiosis. Plant Cell 17, 2217-2229

36 Wegel, E. et al. (1998) Mycorrhiza mutants of Lotus japonicus define genetically independent steps during symbiotic infection. Mol. PlantMicrobe Interact. 11, 933-936

37 Genre, A. et al. (2005) Arbuscular mycorrhizal fungi elicit a novel intracellular apparatus in Medicago truncatula root epidermal cells before infection. Plant Cell 17, 3489-3499

38 Fournier, J. et al. (2008) Mechanism of infection thread elongation in root hairs of Medicago truncatula and dynamic interplay with associated rhizobial colonization. Plant Physiol. 148, 1985-1995
39 Genre, A. et al. (2008) Prepenetration apparatus assembly precedes and predicts the colonization patterns of arbuscular mycorrhizal fungi within the root cortex of both Medicago truncatula and Daucus carota. Plant Cell 20, 1407-1420

40 Feddermann, N. et al. (2010) The PAM1 gene of petunia, required for intracellular accommodation and morphogenesis of arbuscular mycorrhizal fungi, encodes a homologue of VAPYRIN. Plant J. 64, 470-481

41 Pumplin, N. et al. (2010) Medicago truncatula Vapyrin is a novel protein required for arbuscular mycorrhizal symbiosis. Plant J. 61, 482-494

42 Zhang, Q.et al. (2010) Two Medicago truncatula half-ABC transporters are essential for arbuscule development in arbuscular mycorrhizal symbiosis. Plant Cell 22, 1483-1497

43 Feddermann, N. and Reinhardt, D. (2011) Conserved residues in the ankyrin domain of VAPYRIN indicate potential protein-protein interaction surfaces. Plant Signal. Behav. 6 (in press)

44 Michaely, P. et al. (2002) Crystal structure of a 12 ANK repeat stack from human ankyrinR. EMBO J. 21, 6387-6396

45 Hijri, M. and Sanders, I.R. (2005) Low gene copy number shows that arbuscular mycorrhizal fungi inherit genetically different nuclei. Nature 433, 160-163

46 Kuhn, G. et al. (2001) Evidence for the evolution of multiple genomes in arbuscular mycorrhizal fungi. Nature 414, 745-748

47 Pawlowska, T.E. and Taylor, J.W. (2004) Organization of genetic variation in individuals of arbuscular mycorrhizal fungi. Nature 427, 733-737

48 Jany, J.L. and Pawlowska, T.E. (2010) Multinucleate spores contribute to evolutionary longevity of asexual Glomeromycota. Am Nat. 175, 424435

49 Helber, N. and Requena, N. (2008) Expression of the fluorescence markers DsRed and GFP fused to a nuclear localization signal in the arbuscular mycorrhizal fungus Glomus intraradices. New Phytol. $177,537-548$

50 Martin, F. et al. (2008) The long hard road to a completed Glomus intraradices genome. New Phytol. 180, 747-750

51 Chisholm, S.T. et al. (2006) Host-microbe interactions: shaping the evolution of the plant immune response. Cell 124, 803-814

52 Croll, D. et al. (2009) Nonself vegetative fusion and genetic exchange in the arbuscular mycorrhizal fungus Glomus intraradices. New Phytol. 181, 924-937

53 Angelard, C. et al. (2010) Segregation in a mycorrhizal fungus alters rice growth and symbiosis-specific gene transcription. Curr. Biol. 20, 1216-1221

54 Croll, D. and Sanders, I.R. (2009) Recombination in Glomus intraradices, a supposed ancient asexual arbuscular mycorrhizal fungus. BMC Evol. Biol. 9, 13

55 den Bakker, H.C. et al. (2010) Clonality and recombination in the life history of an asexual arbuscular mycorrhizal fungus. Mol. Biol. Evol. 27, 2474-2486

56 Klironomos, J.N. (2003) Variation in plant response to native and exotic arbuscular mycorrhizal fungi. Ecology 84, 2292-2301

57 Schwartz, M.W. and Hoeksema, J.D. (1998) Specialization and resource trade: biological markets as a model of mutualisms. Ecology 79, 1029-1038

58 Herre, E.A. et al. (1999) The evolution of mutualisms: exploring the paths between conflict and cooperation. Trends Ecol. Evol. 14, 49-53

59 Hibbett, D.S. et al. (2000) Evolutionary instability of ectomycorrhizal symbioses in basidiomycetes. Nature 407, 506-508

60 Martin, F. et al. (2008) The genome of Laccaria bicolor provides insights into mycorrhizal symbiosis. Nature 452, 88-92

61 Sachs, J.L. et al. (2010) Origins of cheating and loss of symbiosis in wild Bradyrhizobium. J. Evol. Biol. 23, 1075-1089

62 Singleton, P.W. and Stockinger, K.R. (1983) Compensation against ineffective nodulation in soybean. Crop Sci. 23, 69-72

63 Kiers, E.T. et al. (2003) Host sanctions and the legume-rhizobium mutualism. Nature 425, 78-81

64 Javot, H. et al. (2007) A Medicago truncatula phosphate transporter indispensable for the arbuscular mycorrhizal symbiosis. Proc. Natl. Acad. Sci. U.S.A. 104, 1720-1725

65 Maeda, D. et al. (2006) Knockdown of an arbuscular mycorrhizainducible phosphate transporter gene of Lotus japonicus suppresses mutualistic symbiosis. Plant Cell Physiol. 47, 807-817 
66 Bever, J.D. et al. (2009) Preferential allocation to beneficial symbiont with spatial structure maintains mycorrhizal mutualism. Ecol. Lett. $12,13-21$

67 Dale, C. and Moran, N.A. (2006) Molecular interactions between bacterial symbionts and their hosts. Cell 126, 453-465

68 Judson, O.P. and Normark, B.B. (1996) Ancient asexual scandals. Trends Ecol. Evol. 11, A41-A46

69 de Visser, J. and Elena, S.F. (2007) The evolution of sex: empirical insights into the roles of epistasis and drift. Nat. Rev. Genet. 8, 139-149

70 Schurko, A.M. et al. (2009) Signs of sex: what we know and how we know it. Trends Ecol. Evol. 24, 208-217

71 Giovannetti, M. et al. (1999) Anastomosis formation and nuclear and protoplasmic exchange in arbuscular mycorrhizal fungi. Appl. Environ. Microbiol. 65, 5571-5575

72 García-Garrido, J.M. and Ocampo, J.A. (2002) Regulation of the plant defence response in arbuscular mycorrhizal symbiosis. J. Exp. Bot. 53, 1377-1386

73 Catford, J.G. et al. (2003) Suppression of arbuscular mycorrhizal colonization and nodulation in split-root systems of alfalfa after pre-inoculation and treatment with Nod factors. J. Exp. Bot. 54, 1481-1487
74 Balzergue, C. et al. (2010) The regulation of arbuscular mycorrhizal symbiosis by phosphate in pea involves early and systemic signaling events. J. Exp. Bot. 62, 1049-1060

75 Breuillin, F. et al. (2010) Phosphate systemically inhibits development of arbuscular mycorrhiza in Petunia hybrida and represses genes involved in mycorrhizal functioning. Plant J. 64, 1002-1017

76 van der Heijden, M.G.A. (2004) Arbuscular mycorrhizal fungi as support systems for seedling establishment in grassland. Ecol. Lett. 7, 293-303

77 van der Heijden, M.G.A. and Horton, T.R. (2009) Socialism in soil? The importance of mycorrhizal fungal networks for facilitation in natural ecosystems. J. Ecol. 97, 1139-1150

78 Egerton-Warburton, L.M. et al. (2007) Common mycorrhizal networks provide a potential pathway for the transfer of hydraulically lifted water between plants. J. Exp. Bot. 58, 1473-1483

79 Smith, S.E. et al. (2003) Mycorrhizal fungi can dominate phosphate supply to plants irrespective of growth responses. Plant Physiol. 133, $16-20$

80 Smith, S.E. et al. (2004) Functional diversity in arbuscular mycorrhizal (AM) symbioses: the contribution of the mycorrhizal $\mathrm{P}$ uptake pathway is not correlated with mycorrhizal responses in growth or total $\mathrm{P}$ uptake. New Phytol. 162, 511-524 